\title{
Deep brain stimulation might alleviate parkinsonism by reducing excessive synchronization in primary motor cortex
}

The beneficial effects of deep brain stimulation (DBS) in Parkinson disease (PD) could be attributed to reversal of exaggerated coupling of primary motor cortex activity, according to new research. DBS is widely used to treat PD and, increasingly, other movement and psychiatric disorders, but its mechanism of action has been elusive.

In the healthy brain, the activity of neurons within the primary motor cortex is synchronized. In the circuits that control movement, this activity oscillates at about $20 \mathrm{~Hz}$, which is known as the beta rhythm. Flexible, dynamically modulated rhythmic activity helps co-ordinate communication across brain areas, but in $\mathrm{PD}$, synchronization is excessively high.
Coralie de Hemptinne and colleagues recorded neuronal activity in the primary motor cortex during DBS implantation surgery in 23 patients with PD. Before initiation of basal ganglia DBS, the neurons in the primary motor cortex were strongly coupled to the phase of the beta rhythm. Turning on DBS reduced the excessive locking of neuronal spiking activity in beta oscillations.

"Mechanistically, this reduction could reflect releasing neurons from the inflexible pattern of activity," says de Hemptinne.

In 12 of the patients, the investigators also recorded motor cortex activity during an arm movement task. The patients' parkinsonian motor signs were alleviated on a timescale similar to the DBS-induced decoupling of excessive synchronization. Adjusting DBS according to patients' brain activity could enable individualized treatment and help reduce adverse effects associated with DBS. The researchers are currently testing a new DBS device that can simultaneously detect excessive synchronization and trigger 'on demand' stimulation. According to de Hemptinne, patient-tailored, smart DBS has the potential to soon become reality.

\section{Hemi Malkki}

Original article de Hemptinne, C. et al. Therapeutic deep brain stimulation reduces cortical phase-amplitude coupling in Parkinson's disease. Nat. Neurosci. doi:10.1038/nn.3997 